\title{
Dynamic Three-Dimensional Echocardiographic Imaging of Congenital Heart Defects in Infants and Children by Computer-Controlled Tomographic Parallel Slicing Using a Single Integrated Ultrasound Instrument
}

\author{
DAVID R. FULTON, M.D., GERALD R. MARX, M.D., NATESA G. PANDIAN, M.D., \\ BARBARA A. ROMERO, B.S., BERNARD MUMM, M.S., \\ MICHAEL KRAUSS, HELMUT WOLLSCHLÄGER, M.D., \\ ACHI LUDOMIRSKY, M.D., * and QI-LING CAO, M.D. \\ Division of Pediatric Cardiology, Department of Pediatrics, The Floating Hospital for Infants \\ and Children, Tufts-New England Medical Center and the Cardiovascular Imaging and \\ Hemodynamic Laboratory, Tufts-New England Medical Center, Boston, Massachusetts; *Mott \\ Children's Hospital, University of Michigan, Ann Arbor, Michigan
}

Three-dimensional cardiac reconstruction generated from transesophageal interrogation can be performed using an integrated unit that captures, processes, and postprocesses tomographic parallel slices of the heart. This probe was used for infants and young children in the transthoracic position to evaluate the feasibility of producing three-dimensional cardiac images with capability for realtime dynamic display. Twenty-two infants and children (range 1 day-3.5 years) underwent image acquisition using a $16 \mathrm{~mm} 5 \mathrm{MHz} 64$ element probe placed over the precordium. Two infants were also imaged from the subcostal position. Data was obtained and stored over a single cardiac cycle after acceptable cardiac and respiratory gating intervals were met. The transducer was advanced in 0.5-1 $\mathrm{mm}$ increments over the cardiac structures using identical acquisition criteria. The images were reconstructed from the stored digital cubic format and could be oriented in any desired plane. In 9 of the 22 infants the images obtained were of optimal quality. The images obtained displayed normal cardiac structures emphasizing depth relationships as well as visualization of planes not generally demonstrated by two-dimensional imaging. Several lesions were also depicted in a unique fashion using this technique. Though the method employed was limited by movement artifact and reconstruction time, the quality of the three-dimensional display was excellent and enhanced by realtime demonstration. The transthoracic approach was successful in capturing sufficient data to create three-dimensional images, which may have further application in more accurate diagnosis of complex cardiac abnormalities and generation of planes of view which could duplicate surgical visualization of a lesion. Further assessment of the technique in infants with congenital heart disease is warranted. (ECHOCARDIOGRAPHY, Volume 11, March 1994)

echocardiography, three-dimensional echocardiography, three-dimensional reconstruction, ultrasound, congenital heart disease

Address for correspondence and reprints: David R. Fulton, M.D., Division of Pediatric Cardiology, Tufts New England Medical Center, Box 171, 750 Washington Street, Boston, MA 02111. Fax: 617-350-8376.
Two-dimensional echocardiography has become an invaluable tool in the diagnosis and management of congenital heart disease. It is capable of providing detailed images of most 
defects with the effect enhanced by real-time display. However, since the images obtained have only two-dimensional spatial representation, the echocardiographer must create a mental three-dimensional perspective of the image. Technology that could bridge this three-dimensional spatial gap would advance imaging significantly.

Multiple attempts have been made to obtain three-dimensional cardiac images in adults using various modalities. ${ }^{1-20}$ However, such techniques as ultrafast computerized tomography and cardiac magnetic resonance imaging are promising but require prolonged acquisition time, limiting temporal resolution of the moving heart and are less accessible to the cardiologist than conventional two-dimensional echocardiography. Manual or automatic echocardiographic border detection methods can derive volumetric data, but complete reconstruction of the heart has not been possible. Further, intravascular ultrasound can provide three-dimensional images of vessels, but not intracardiac structures. All of these methods are further impeded by the need for offline analysis using independent computer configuration.

Recently, a prototype echocardiographic unit has become available using a transesophageal probe with a sliding movable transducer. This transducer is advanced over the length of the heart at a prescribed rate and distance to obtain multiple, parallel tomographic echocardiographic slices of the heart throughout the cardiac cycle. ${ }^{21}$ The data obtained is fully integrated with a computer used to store, analyze, and manipulate data to form three-dimensional constructs of the heart. Though the intended application in adults is for transesophageal use, the $16 \mathrm{~mm}$ diameter of the probe negates the same method in infants and children. However, the inclusive aspects of the total system led us to attempt to use this same probe in infants and children via a transthoracic window. The purpose of this report is to present our initial experience with the feasibility of data acquisition and manipulation to form three-dimensional constructs of the heart in a young population.

\section{Methods}

\section{Instrumentation}

The echocardiographic unit employed (TomTec, GmbH, Munich, Germany) is capable of standard two-dimensional imaging as well as pulsed wave, continuous wave, and color flow Doppler interrogation. The imaging probe consists of a $5 \mathrm{MHz} 64$ element phased array transducer, $16 \mathrm{~mm}$ in diameter. The transducer is mounted on a movable carriage situated within a housing formed by a series of connected semicircular plastic units. The flexible state of the probe is intended to permit transesophageal introduction of the unit. After appropriate positioning the unit is straightened by means of a cable system strung through the plastic connectors. Water is introduced between the probe cover and a latex sheath covering the transducer used to seal the system to maximize the acoustic properties for ultrasound transmission. In our subjects the probe was used in its fully extended rigid form, positioned as described above.

Electrocardiographic leads and chest wall sensors were placed on the precordium to permit gating of image acquisition to the cardiac and respiratory cycles (Fig. 1A). An "observation period" of 1-2 minutes determined a mean $R R$ interval for the individual subject. Ranges (mean $\pm 40 \mathrm{msec}$ ) were set as acceptable gating intervals for the cardiac cycle. The chest wall sensors measuring thoracic impedance assessed end-expiration. The transducer was advanced to the most distal position within the probe. Image acquisition was initiated throughout the cardiac cycle and if gating criteria were met ( $R R$ interval limits as defined and end-expiration) the set of images for that cycle was stored in the computer (Fig. 1B). The cable motor was activated moving the transducer along a linear direction to a position $0.5-1 \mathrm{~mm}$ proximal to the preceding position to obtain the next tomographic slice (Fig. 1C). The sequence was repeated until approximately 120 slices of $1 \mathrm{~mm}$ thickness were obtained each one full cardiac cycle in duration. In cardiac cycles where either ECG or respiratory gating fell outside predetermined boundaries, the cycle was repeated until the required conditions were met 

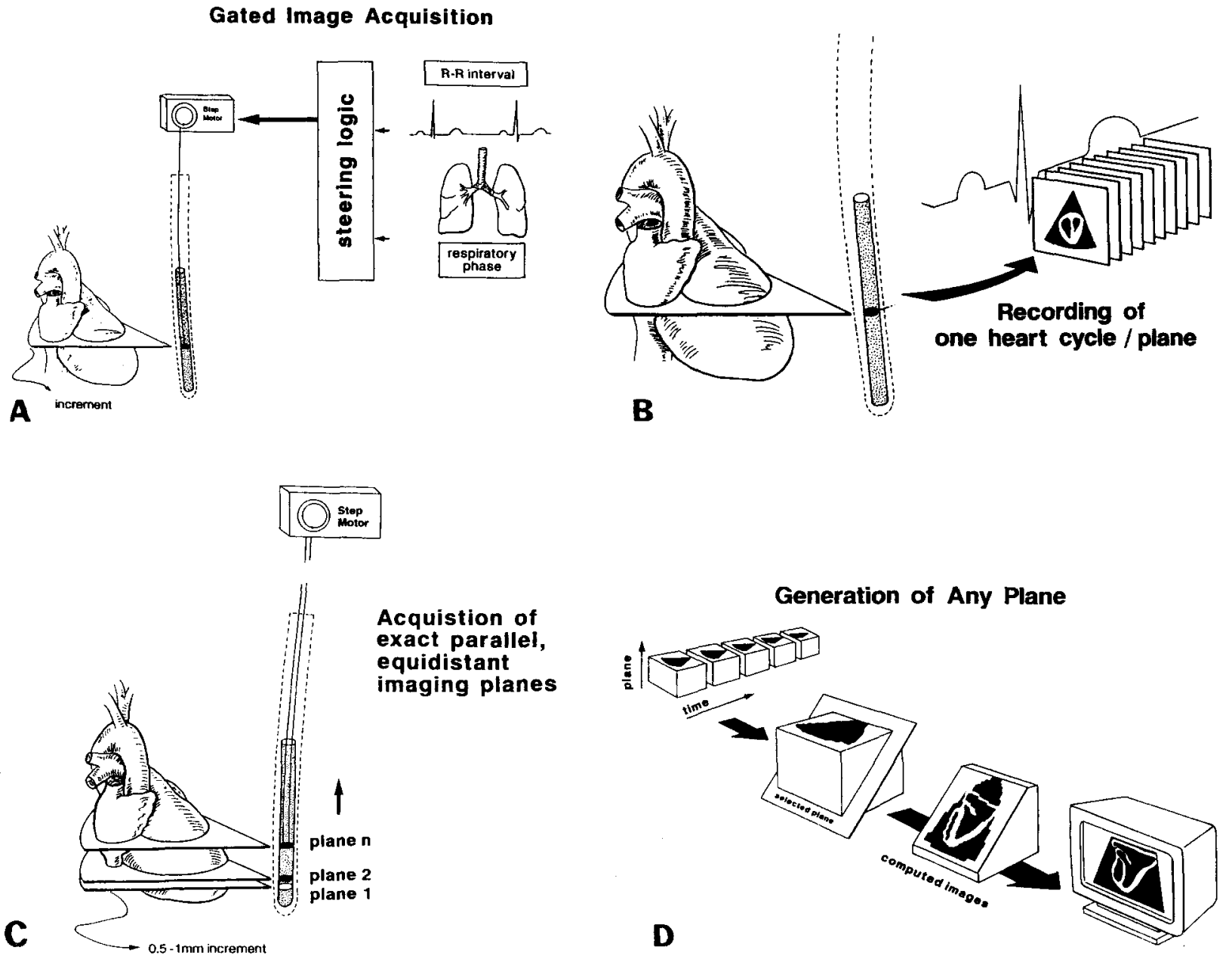

Figure 1. ECG and chest wall sensors gate image acquisition (A) and interface to a step motor that moves the transducer to the next level. A single cardiac cycle of images is acquired at each level $(B)$ while the transducer continues to move through multiple parallel equidistant planes (C) to permit accurate registration of cardiac cycles. Following storage in digital format, any plane of interest can be selected for reconstruction and display of the image (D).

and the frames were accepted for storage. The time required for acquisition was 3-4 minutes.

Each data set was stored and registered in digital format incorporating a matrix $256 \times 128 \times \mathrm{N}(\mathrm{N}=$ number of slices $)$ so that spatial and temporal resolution was achieved with equidistant parallel images (Fig. 1D). The data was stored on a $48633 \mathrm{MHz}$ personal computer with $64 \mathrm{MB}$ RAM and a hard drive capacity of $420 \mathrm{MB}$. Permanent data archiving was made on removable $600 \mathrm{MB}$ laser cartridges capable of accommodating approximately 20 sep- arate patient studies. Raw digital data could be recalled for analysis at any time on or off line.

To create a three-dimensional image, the two-dimensional image was displayed and reviewed. At any desired frame, six cursors could be superimposed over the image in any orientation to create a panel of six individual two-dimensional images. Each image could be played in loop format of one complete cardiac cycle. The images could be rotated in any $\mathrm{X}, \mathrm{Y}$, or $\mathrm{Z}$ plane using the console controls. When the desired frame and position of any image was 
obtained, computer reconstruction of three-dimensional format was initiated. For rapid assessment purposes, every second voxel was analyzed for gray scale data sacrificing resolution, but permitting reconstruction time of $7-8$ minutes.

Final reconstruction was accomplished by analysis of all voxel information in the data cube. To achieve three-dimensional effects, gray scale attributes were assigned according to algorithms for distance shading. ${ }^{22,23}$ The gray scale representation in distance shading is inversely related to the viewpoint of any given structure. In general, near field structures appear opaque or brighter, while distant regions are darker shades of gray or transparent. Completion of this format required approximately 60 minutes. The reconstructed freeze-frame image could be played in loop format over the whole cardiac cycle. The computer memory was sufficient to enable simultaneous reconstruction of three separate three-dimensional images rotated about the transverse plane.

\section{Subjects}

After obtaining informed consent, 22 infants and children, median age 8 months (range 1 day-3.5 years), underwent transthoracic or subcostal echocardiographic examination (Table I). When necessary, a single dose of chloral hydrate $50-100 \mathrm{mg} / \mathrm{kg}$ p.o. was administered to minimize superfluous movement. The echocardiographer positioned the probe over the precordium from the apex to the base in an effort to maximize contact of the rigid probe with the chest wall. To guard against movement during acquisition, the probe required stabilization using one hand positioned at either end. In two infants, subcostal imaging was performed with the probe parallel to the diaphragm. The examinations were completed without complication.

\section{Results}

Of the 22 patients evaluated, only nine had studies that had adequate data suitable for three-dimensional reconstruction. These limitations were the result of transducer movement

\begin{tabular}{|c|c|c|c|}
\hline \multicolumn{4}{|c|}{$\begin{array}{c}\text { TABLE I } \\
\text { Patient Population }\end{array}$} \\
\hline Diagnosis & & Age & $\begin{array}{c}\text { 3-D Echo } \\
\text { Quality } \\
\{\text { Scale 1-4\} }\end{array}$ \\
\hline 1. ASD Secundum & 1 & Day & \\
\hline 2. D-TGA, IVS & 1 & Day & 3 \\
\hline 3. ASD, Coarctation & 15 & Days & \\
\hline 4. P.S. Valvar & 1 & Month & 2 \\
\hline 5. TOF & 2 & Months & \\
\hline 6. Coarctation & & 5 Months & \\
\hline 7. CCAVC & 3 & Months & 3 \\
\hline 8. CCAVC & 5 & Months & 3 \\
\hline 9. VSD - ASD & 5 & Months & 4 \\
\hline 10. Normal & 5 & Months & 3 \\
\hline 11. TOF & 8 & Months & \\
\hline 12. TOF & 8 & Months & \\
\hline 13. RV Tumor & 10 & Months & 3 \\
\hline 14. Normal & 1 & Year & \\
\hline 15. Single ventricle & 14 & Months & 2 \\
\hline 16. P.S. Valvar & 15 & Months & \\
\hline 17. Normal & 15 & Months & \\
\hline 18. Normal & 2 & Years & \\
\hline 19. TOF, PA & & 5 Years & \\
\hline 20. VSD & & Years & \\
\hline $\begin{array}{l}\text { 21. ASD, Sinus } \\
\text { venosus }\end{array}$ & & 5 Years & 4 \\
\hline
\end{tabular}

ASD $=$ atrial septal defect; D-TGA $=$ D-transposition of the great arteris; IVS = intact ventricular septum; PS = pulmonic stenosis; TOF = tetralogy of fallot; CCAVC = complete common AV canal; VSD = ventricular septal defect; PA = pulmonary atresia.

or movement of the infant interfering with the acquisition of parallel linear images. Though the two-dimensional images may have appeared satisfactory on line, three-dimensional reconstruction proved futile. Of the nine who had optimal data, the three-dimensional formats were striking and several are illustrated in the following freeze-frame examples.

The first image is obtained from an infant with an atrial septal defect. The figure is rotated in $60^{\circ}$ angled increments from left axial oblique to right axial oblique. All images are shown in diastole. The far left anterior oblique (LAO) view (Fig. 2A) shows the left ventricular cavity, particularly the left septal surface. The mitral valve leaflets and papillary muscles are anterior in this cut and shaded brightly. The posterior left atrial wall is darker and a pulmo- 

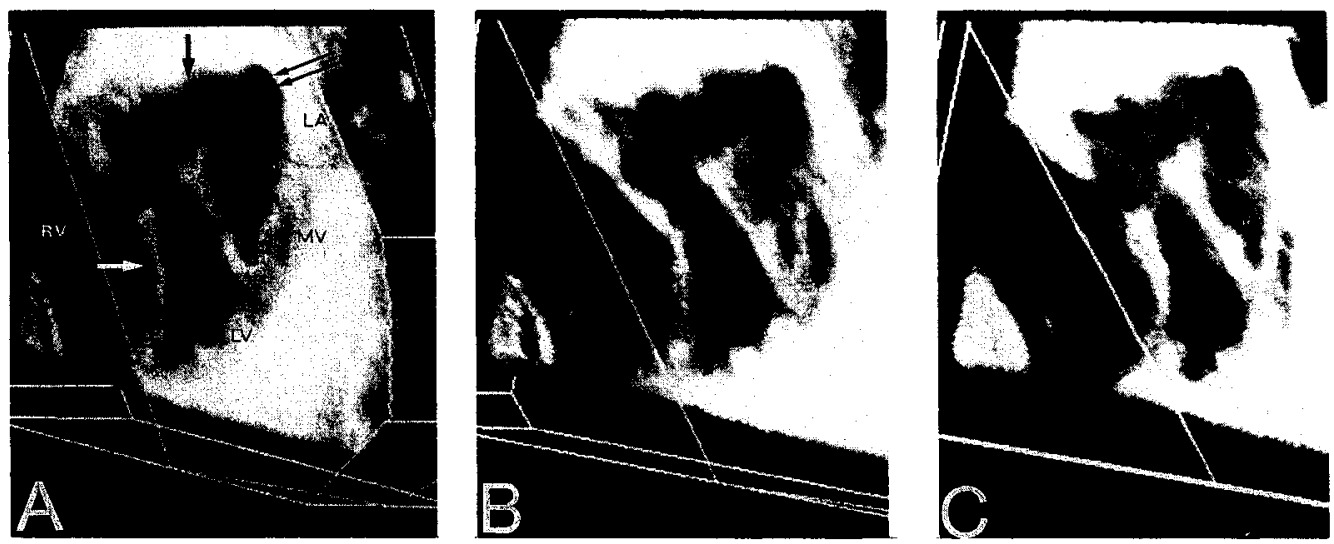

Figure 2. Three-dimensional reconstruction of the heart in an infant with an atrial septal defect rotated in $60^{\circ}$ increments. From the LAO view (A), atrial defect is noted (black arrow) with ventricular septum seen in depth (white arrow). Double arrows highlight single pulmonary vein with multiple veins seen in posterior LA wall in subsequent images. (LAO = left anterior oblique; $R V=$ right ventricle; $L V=$ left ventricle; $L A=$ left atrium; $M V=$ mitral valve).

nary vein is barely visible in the superior posterior left atrial cavity. After rotating $60^{\circ}$ (Fig. 2B) the posterior left ventricular cavity is best visualized while the ventricular septum and left ventricular free wall are seen nearly on end. The left ventricular outflow tract is unobstructed though the aortic valve leaflets are not well visualized. The supravalvar region of the ascending aorta can be tracked superiorly for a short distance before its course is lost in this cut. The right superior and inferior pulmonary veins are seen from this angle. In the final image, rotated an additional $60^{\circ}$ (Fig. $2 \mathrm{C}$ ), the right ventricle and septal surface are well seen. The aortic valve leaflets are noted in the closed position. The left superior pulmonary vein is now seen lateral to the right upper pulmonary vein.

The technique is versatile in that it permits cuts through any plane of the heart as demonstrated in the next image (Fig. 3A Diastole; Fig. $3 \mathrm{~B}$ Systole). This 10-month-old infant with tuberous sclerosis has an associated intracardiac rhabdomyoma. The tumor is attached to the right ventricular septal surface and is somewhat irregular, but the tissue characteristics of the mass can be readily distinguished from those of the septal surface. The points of attach- ment are well discerned (small arrows). From this right axial oblique cut the left ventricular endocardial surface is well portrayed from the free lateral wall (lighter) to the posterior surface (darker). In real-time display these differences are enhanced and progressive changes in left ventricular wall thickness are well visualized.

The opportunity to view images from planes not previously available is demonstrated in the next example (Fig. 4). This is made just above the plane of the atrioventricular (AV) valves in an infant with a structurally normal heart. In the still frame the anterior and posterior leaflets of the mitral valve are noted in diastole. The left ventricular inflow region is inferior to the leaflets, but little detail of the endocardial surface can be seen. The tricuspid valve is open wide so the leaflets are not seen in this cut, but the right ventricular surface is seen below the level of the AV valve plane. The crest of the ventricular septum separates the $A V$ valves centrally. Using dynamic (four-dimensional) display, the motion of the valve leaflets through the cardiac cycle is depicted from coaptation of the free edges of the leaflets to the widely separated diastole position.

The utility of the system for congenital de- 

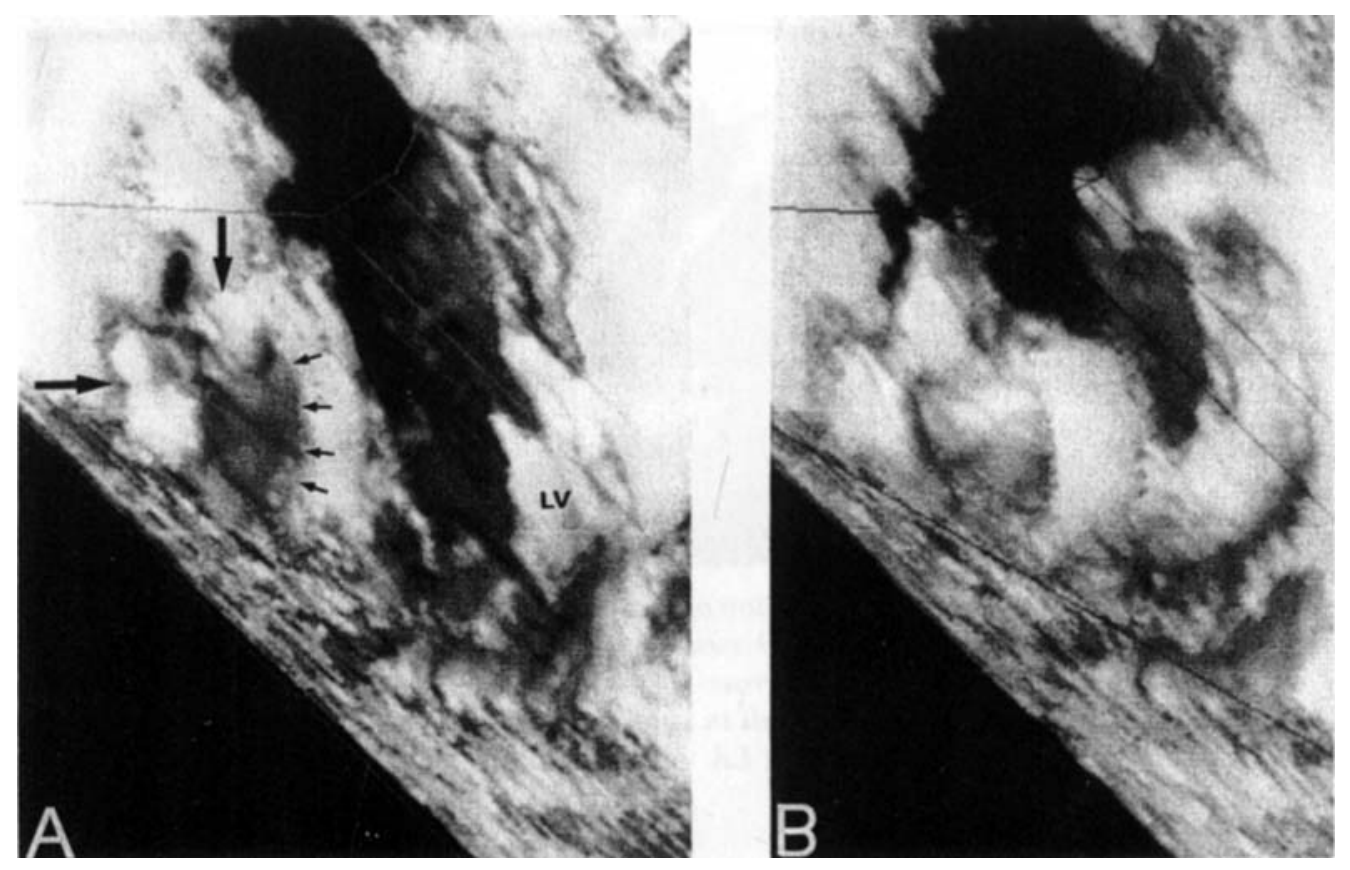

Figure 3. Three-dimensional image of 10-month-old male infant with rhabdomyoma attached to the right ventricular septal surface (small arrows). The large arrows delineate the superior and lateral margin of the tumor. The $L V$ cavity is seen extending posteriorly in diastolic frame (A), and foreshortened in the systolic frame (B). $L V=$ left ventricle.

fects is depicted in the final figure (Figs. 5A and $5 \mathrm{~B}$ ) that illustrates the heart of an infant with a complete AV canal showing an ASD, ostium primum type (white arrow), and a VSD of the AV canal type (black arrow). The VSD can be seen again by rotating the image and creating a slice through the plane of the tricuspid valve in a plane not achievable by two-dimensional echocardiography. In real-time, the leaflets of the valve are fully visualized in systole.

\section{Discussion}

This study demonstrates for the first time that dynamic three-dimensional reconstruction of a beating heart is feasible using a transthoracic approach with phased array ultrasound imaging. The probe implemented in this study is used in adults as a transesophageal instrument, conceived to circumvent the limited window frequently encountered in such subjects. The size of the gastroscope limits its use for pe- diatric patients undergoing transesophageal studies, especially younger children and infants. As a consequence, the $5 \mathrm{MHz}$ probe was used as a conventional transthoracic unit and succeeded in generating high quality two-dimensional images. Gating with the ECG and respiratory cycle permitted acquisition of successive slices corrected for the cardiac cycle and chest wall movement. Equidistant parallel slices over a $12 \mathrm{~cm}$ distance were facilitated by the motorized gantry system with low tolerance for deviation from preset boundaries. Since the transducer could not be advanced to the next level of the heart unless the spatial and temporal limitations were adhered to tightly, there was some concern that the overall acquisition time would be unwieldy and that, as a result, relaxation of preset controls for position and time would be necessary, limiting ultimate resolution of the three-dimensional reconstruction. However, acquisition times proved to be similar to those achieved in adults undergoing 


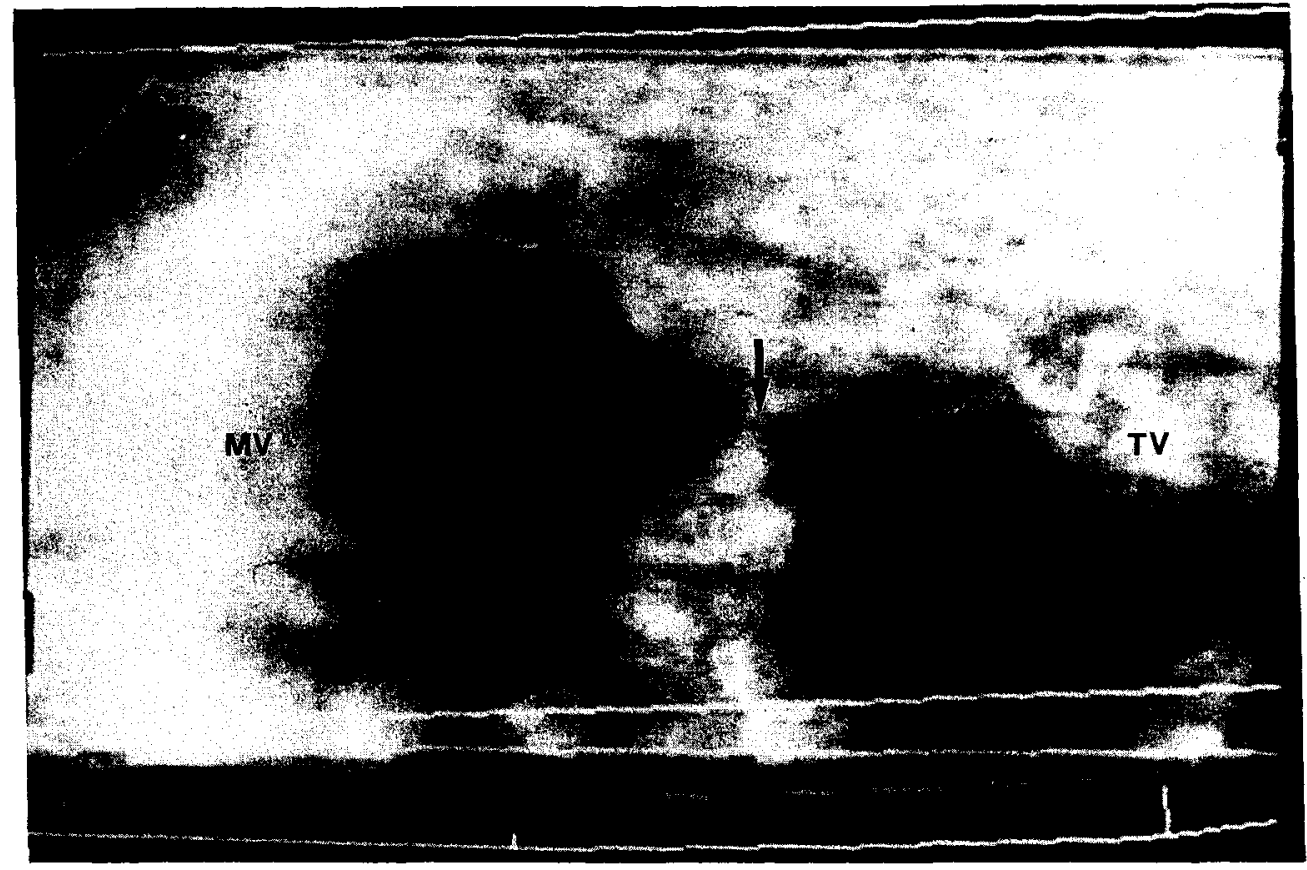

Figure 4. Atrio-ventricular valves visualized from a superior and slightly posterior plane in a normal heart. The MV leaflets are seen in diastole while the TV leaflets are widely separated, exposing the $R V$ endocardial surface. The crest of the ventricular septum is noted by the arrow. $M V=$ mitral valve; $T V=$ tricuspid valve; $R V=$ right ventricle.

transesophageal application of the device. We decreased acquisition times even further by initiating frame capture at the inferior boundary of the heart rather than at the distal end of the probe.

Though our overall study group is small, the three-dimensional images were able to detail several congenital abnormalities. Given the infinite number of planes available for scrutiny, these defects could be visualized by conventional display or from novel directions. The wide array of presentation has several advantages. In this initial effort, images in this study were obtained, reviewed, and reprocessed to portray normal cardiac anatomy, and by extension, the size, shape, and location of defects in the atrial or ventricular septum, attachment and movement of the AV valves, and size, position, and adherence of intracardiac masses. Though outside the scope of this study, other applications appear readily feasible including aggregate volumetric measurements of cardiac chambers, quantitative determination of ventricular wall mass and volume of pericardial effusion. Specific investigation is necessary, however, to determine the accuracy of the proposed measurements. In a somewhat more farreaching application, it may be possible to superimpose a planned surgical intervention on this three-dimensional model to anticipate the benefits or limitations of a particular approach prior to the actual surgery. Intraoperative transesophageal assessment using three-dimensional reconstruction could confirm the efficacy of repair, particularly in complex lesions.

Several limitations were encountered that need to be considered. The contour of the chest wall in this population produced less than optimal contact surface for this rigid, linear transducer probe, resulting in several studies in which three-dimensional reconstruction could not be achieved. A flexible probe that could be 

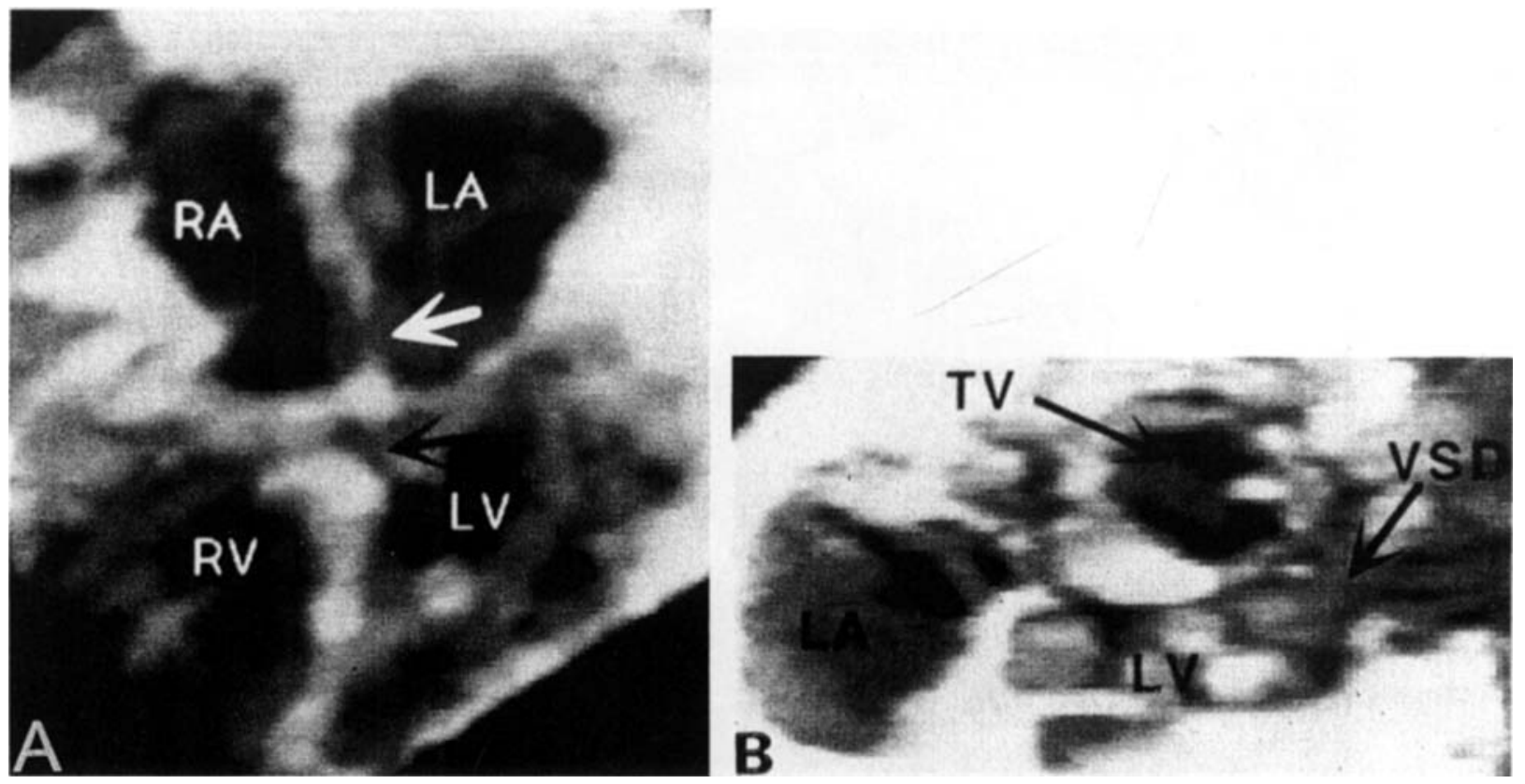

Figure 5. Infant with a complete AV canal (A) showing both the atrial (white arrow) and ventricular defect (black arrow). Rotation of the image and slicing through plane of tricuspid valve components (B) showing the VSD in a novel imaging orientation not achieved by two-dimensional echo. $R A=$ right atrium; $L A=$ left atrium; $R V=$ right ventricle; $L V=$ left ventricle; $V S D=$ ventricular septal defect; $A S D=$ atrial septal defect; $T V=$ tricuspid valve.

applied for continuous contact along the complete imaging axis of the heart would conceivably result in improved resolution, particularly at the apex and base of the heart and by extension to the great vessels. However, a curvilinear design might not be accomplished without sacrificing the equidistant parallel slices now obtained, requiring correction for a curved pathway, which allows smooth transition at 1 $\mathrm{mm}$ increments. Longer acquisition time exchanged for higher quality images would be acceptable; however, it would then be preferable to devise a method to stabilize the probe in precise location eliminating the possibility that a hand-held probe would move, inadvertently sacrificing the quality of the data, another source of suboptimal three-dimensional reconstruction. Most important, the clinical applicability of this technology will most likely be influenced not only by the quality of the image, but also by the time required for reconstruction, display, and storage of the desired images. Currently, three-dimensional reconstruction from a single selected two-dimensional plane takes 6-7 minutes. Should the reconstruction fail to display the anticipated image, another plane must be selected and the time is duplicated. Clearly, the infinite number of planes achievable is an advantage; however, the time factor must be set against the array of possible images. It is conceivable that certain key views will emerge with greater use, and these could be formatted as default images with reconstruction centered around them; fine alterations could be made for more complex lesions or simple lesions with specific queries. Some of these hurdles will be cleared by faster computer processing. A smaller gastroscope design utilizing the same transducer frequency would permit intraoperative application in smaller patients than adults, thereby broadening the range of potential subjects. 


\section{Conclusion}

In conclusion, this study has shown the feasibility of three-dimensional and four-dimensional tomographic images in infants and young children with congenital heart abnormalities utilizing a reproducible transthoracic approach. Though some temporal and spatial obstacles remain as impediments to widespread and comprehensive utilization, when overcome, this modality should find wide acceptance for surgical decision making, as a teaching tool, and for previously elusive quantitative measurements.

Acknowledgment: The authors wish to thank Julianne Foley for her assistance in the preparation of this manuscript.

\section{References}

1. Dekker DL, Piziali RL, Dong E: A system for ultrasonically imaging the human heart in three dimensions. Comput Biomed Res 1974;7: 544-553.

2. Kuroda T, Kinter TM, Seward JB, et al: Accuracy of three-dimensional volume measurement using biplane transesophageal echocardiographic probe: In vitro experiment. $J A m$ Soc Echocardiogr 1991;4:475-484.

3. Martin RW, Bashein G: Measurement of stroke volume with three-dimensional transesophageal ultrasonic scanning: Comparison with thermodilution measurement. Anesthesiology 1989;70:470-476.

4. Moritz WE, Pearlman AS, McCabe DH, et al: An ultrasonic technique for imaging the ventricle in three dimensions and calculating its volume. IEEE Trans Biomed Eng 1983;30: 482-491.

5. Geiser EA, Lupkiewicz SM, Christie LG, et al: A framework for three-dimensional time-varying reconstruction of the human left ventricle: Sources of error and estimation of their magnitude. Comput Biomed Res 1980;13:225241.

6. Ghosh A, Nanda NC, Maurer G: Three-dimensional reconstruction of echocardiographic images using the rotation method. Ultrasound Med Biol 1982;8:655-661.

7. Nikravesh PE, Skorton DJ, Chandran KB, et al: Computerized three-dimensional finite ele- ment reconstruction of the left ventricle from cross-sectional echocardiograms. Ultrasonic Imaging 1984;6:48-59.

8. Levine RG, Handschumacher MD, Sanfilippo AJ, et al: Three-dimensional echocardiographic reconstruction of the mitral valve, with implications for the diagnosis of mitral valve prolapse. Circulation 1989;80:589-598.

9. Raichlen JS, Trivedi SS, Herman GT, et al: Dynamic three-dimensional reconstruction of the left ventricle from two-dimensional echocardiograms. JACC 1986;8:364-370.

10. Rosenfield K, Losordo DW, Ramaswamy K, et al: Three-dimensional reconstruction of human coronary and peripheral arteries from images recorded during two-dimensional intravascular ultrasound examination. Circulation 1991;84: 1938-1956.

11. Pini R, Monnini E, Masotti L, et al: Echocardiographic three-dimensional visualization of the heart. NATO ASI Series 1990;60:263274.

12. McCann HA, Chandrasekaran $\mathrm{K}$, Hoffman EA, et al: A method for three-dimensional ultrasonic imaging of the heart in vivo. Dynamic Cardiovascular Imaging 1987;1:97-109.

13. Matsumoto M, Matsuo $\mathrm{H}$, Kitabatake $\mathrm{A}$, et al: Three-dimensional echocardiograms and twodimensional echocardiographic images at desired planes by a computerized system. Ultrasound Med Biol 1977;3:163-178.

14. Raqueno R, Ghosh A, Nanda NC, et al: Fourdimensional reconstruction of two-dimensional echocardiographic images. Echocardiography 1989;6:323-337.

15. Sheikh KH, Smith SW, Von Ramm 0, et al: Real-time, three-dimensional echocardiography: Feasibility and initial use. Echocardiography 1991;8:119-125.

16. Herman GT, Liu HK: Display of three-dimensional information in computed tomography. $J$ Comput Assist Tomogr 1977;1:155.

17. Feiring AJ, Rumberger JA, Reiter SJ, et al: Determination of left ventricular mass in dogs with rapid-acquisition cardiac computed tomographic scanning. Circulation 1985;72: $1355-1364$.

18. Reiter SJ, Rumberger JA, Feiring AJ, et al: Precision of measurements of right and left ventricular volume by cine computed tomography. Circulation 1986;74:890-900.

19. Axel L, Herman GT, Udupa JK, et al: Three dimensional display of nuclear magnetic reso- 
nance (NMR) cardiovascular images. $J$ Comput Assist Tomogr 1983;7:172-174.

20. Laschinger JC, Vannier MW, Gronemeyer S, et al: Noninvasive three-dimensional reconstruction of the heart and great vessels by ECGgated magnetic resonance imaging: A new diagnostic modality. Ann Thorac Surg 1988;45: 505-514.

21. Wollschläger $\mathbf{H}$, Zeiher $\mathrm{AM}$, Klein $\mathrm{HP}$, et al: Transesophageal echo computer tomography: A new method for dynamic 3-D imaging of the heart. Circulation 1989:II-569.

22. Hoehne KH, Delapaz RL, Bernstein R, et al: Combined surface display and reformatting for the three-dimensional analysis of tomographic data. Invest Radiol 1987;22:658-664.

23. Hoehne KH, Bomans M, Pommert A, et al: 3D visualization of tomographic volume data using the generalized voxel model. Visual Computer 1990;6:28-35. 